\title{
Structure Generation and Analysis from the Loosest to the Densest Random Packings
}

\author{
Gary W. Delaney ${ }^{1, \star}$ \\ ${ }^{1}$ CSIRO Data61, Private Bag 10, Clayton South, VIC 3168, Australia
}

\begin{abstract}
We explore sets of jammed packings of frictional spheres and ellipsoids generated using the Discrete Element Method. A novel preparation method is presented and analysed, where the internal structure of the system is modified by iteratively removing grains to generate a continuous variation in packing fraction from the densest to the loosest mechanically stable systems. We present a number of measures quantifying the variation in the structure of the system as grains are removed, including the variation in packing fraction, distribution of grain contact numbers, degree of orientational ordering and the degree of displacements of the grains due to rearrangements to achieve mechanical stability after a grain is removed.
\end{abstract}

\section{Introduction}

Random packings of granular particles occur in abundance in natural and industrial systems [1-4]. One of the simplest ways to form a granular packing is to pour a set of particles into a container. This technique can lead to a broad range of densities being obtained depending on the details of the particle shape, the inter-particle friction, the viscosity of the fluid/gas of the containing medium and the fine details of the pouring setup.

In this paper, we will explore the transition between the loose and dense limits of the possible mechanically stable random granular packings. We will do this by employing a novel technique in which we iteratively remove grains and measure the response of the system in terms of the structural rearrangements and the resulting variation in density. We will consider two systems, the first composed of perfectly spherical grains and the second composed of prolate ellipsoids with an aspect ratio of 0.7 .

\section{Computational Model}

We employ the Discrete Element Method (DEM) and in our implementation represent particles as superellipsoids $[5,6]$. The normal force between two contacting particles is given by:

$$
F_{n}=-k_{n} \xi_{n}+C_{n} v_{n},
$$

where $k_{n}$ is a spring constant determining the stiffness of the particles, $\xi_{n}$ is the linear overlap of the particles, $v_{n}$ is the relative normal velocity and $C_{n}$ is a constant related to the coefficient of restitution. The tangential force is given by:

$$
F_{t}=\min \left\{\mu F_{n}, k_{t} \sum v_{t} d t+C_{t} v_{t}\right\}
$$

\footnotetext{
^e-mail: gary.delaney@csiro.au
}
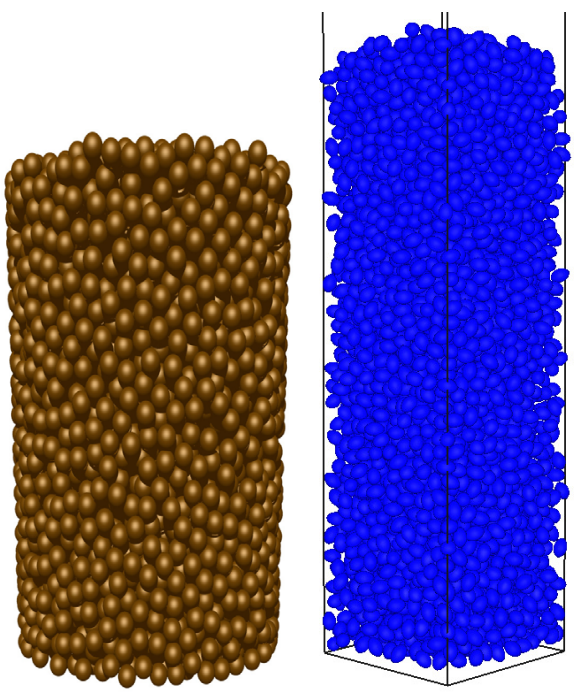

Figure 1. DEM simulation of a packing of (left) 5000 spheres and (right) 4500 prolate ellipsoids with aspect ratio of 0.7 .

where the force vector $F_{t}$ and velocity $v_{t}$ are defined in the plane tangent to the surface at the contact point, and the summation is performed over the duration of the contact [6-8]. The total tangential force, $F_{t}$, is limited by the maximum Coulomb friction $\mu F_{n}$, at which point the surface contact shears and the particles begin to slide over one another. The grains are sedimented in a viscous fluid, where the fluid is modeled using the Stokes equations as described in [9].

Figure 2 shows the packing fraction variation with aspect ratio for systems of mono-disperse ellipsoids with two of their axes equal in length. The parameters used for the simulation are $k_{n}=10^{5} \mathrm{~N} / \mathrm{m}$, a restitution coefficient of $e=0.5$, a particle density $D=2.7 \times 10^{3} \mathrm{~kg} / \mathrm{m}^{3}$ and values 


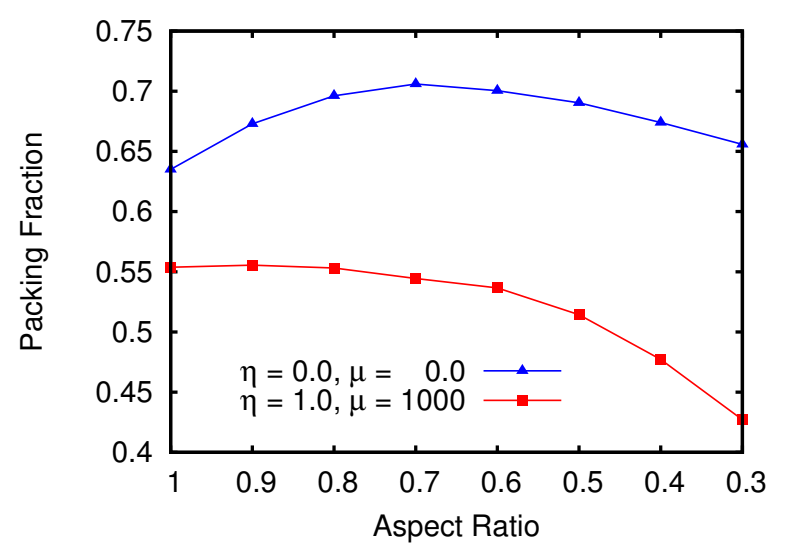

Figure 2. Packing fraction variation with aspect ratio for the densest $(\eta=0.0, \mu=0.0)$ and loosest $(\eta=1.0, \mu=1000)$ ellipsoid packings formed via DEM simulations of a sedimentation process.

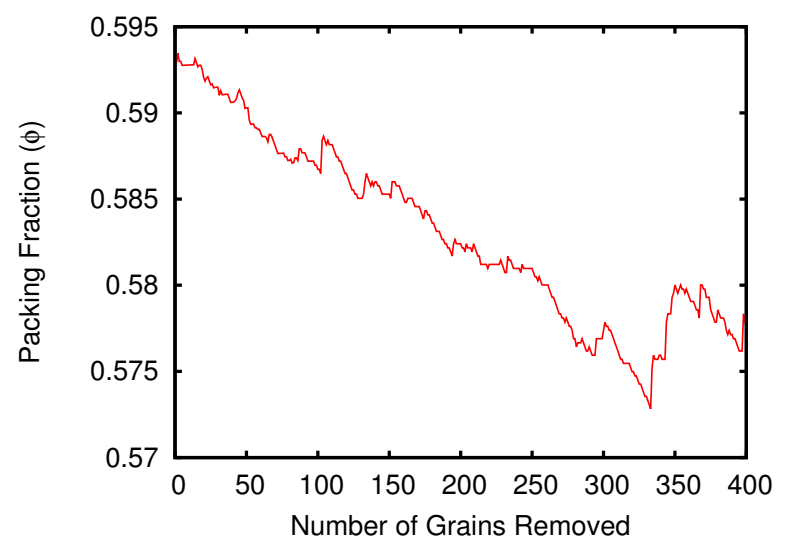

Figure 3. Evolution of the packing fraction as grains are iteratively removed from packing of spheres.

of inter-particle friction ranging from $\mu=0$ to 1000. Values of viscosity ranging from $\eta=0 \mathrm{~Pa}$ s to $\eta=1 \mathrm{~Pa}$ are examined. The fluid has a density $\rho=10^{3} \mathrm{~kg} / \mathrm{m}^{3}$, giving an effective gravity for the particles in the fluid of $0.69 \mathrm{~g}$. We show data for two cases, chosen to demonstrate the extremes of density that are obtainable using this preparation technique. The first case corresponds to zero inter-grain friction $(\mu=0)$ without a background fluid $(\eta=0)$ which produces the densest packings and the second is in the high friction limit with an inter-grain friction $(\mu=1000)$ and a high liquid viscosity ( $\eta=1.0 \mathrm{~Pa} \mathrm{~s}$ ). For dense packings created with zero inter-grain friction, the packing fraction for spheres $(\alpha=1.0)$ is close to the random close packing limit (RCP) value of $\phi=0.64$. As the aspect ratio is varied from unity, we obtain a peak in packing fraction at an aspect ratio of around 0.7 followed by a near linear decline. For the loose packings prepared with high interparticle friction $(\mu=1000)$ in a high viscosity fluid $(\eta=1.0$ $\mathrm{Pa} \mathrm{s}$ ), we obtain packing fractions similar to those measured for the random loose packing limit for spheres at $\phi \simeq 0.55$. As the aspect ratio is varied from unity, we see an initial plateau and then a steady decline between 0.8 and 0.6 followed by a faster rate of decrease. These results are similar to those seen for prolate ellipsoids packings prepared by the same technique [9] and also for random close packings of ellipsoids prepared by a particle expansion technique [1].

Having established the density limits that are obtainable by utilising a pouring (or sedimentation) technique in the previous section, we will now consider a more novel way of transitioning among states in between the loosest and densest mechanically stable packings (the area between the blue and red lines in Figure 3). Here we will constrain our consideration to two systems. The first is composed of 5000 spherical particles in a cylindrical container. Spheres have a packing fraction range for mechanically stable random packings between approximately $\phi=0.55$ and $\phi=0.64$ as shown in Figure 2. The second is a packing of 4500 prolate ellipsoids with aspect ratio 0.7 contained within a periodic box in the $\mathrm{x}$ and $\mathrm{y}$ direction (the 2 directions normal to gravity). These ellipsoids have a packing fraction range for mechanically stable random packings between approximately $\phi=0.545$ and $\phi=0.71$.

To study the transition between different mechanically stable structures, we will employ a simple technique of iteratively removing a single grain chosen at random from the packing and allowing the system to relax to a new mechanically stable state. The inter-particle friction is set to $\mu=1000$ to put us in the infinite friction limit, which allows for the potential to obtain any state between the densest and loosest mechanically stable limits, and the simulation has no background fluid present $(\eta=0)$. Depending on the grain chosen, its removal may lead to anything from no change in the positions of the remaining grains (as the system is still mechanically stable without the removed grain), up to very large scale global rearrangements of the packing as it finds a new mechanically stable state.

Figure 3 shows the evolution of the packing fraction $\phi$ with the number of grains removed for the packing of spherical grains. In this case, we initially start at a packing fraction of $\phi=0.593$, near to the middle of the range of mechanically stable packing fractions that can be obtained for random packings of spheres. As we iteratively remove grains from the system, the packing fraction shows a clear downward trend, with occasional avalanche like events that cause a temporary increase in the packing fraction. These events are caused by removing grains that were playing a particularly important role in the mechanical stability of the system, where their removal causes a large scale rearrangement of the grains and densification of the system under gravity. The increases in packing fraction at these avalanche events is generally only up of a maximum increase of $\Delta \phi=0.002$ until we get to looser packings below $\phi=0.575$ after approximately 300 grains have been removed. At these low densities the system has become much more susceptible to the further removal of grains and we see a more rapid increase in density over successive grain removals from the 330th to the 350th grain removals, where an increase of $\Delta \phi=0.007$ is observed. 


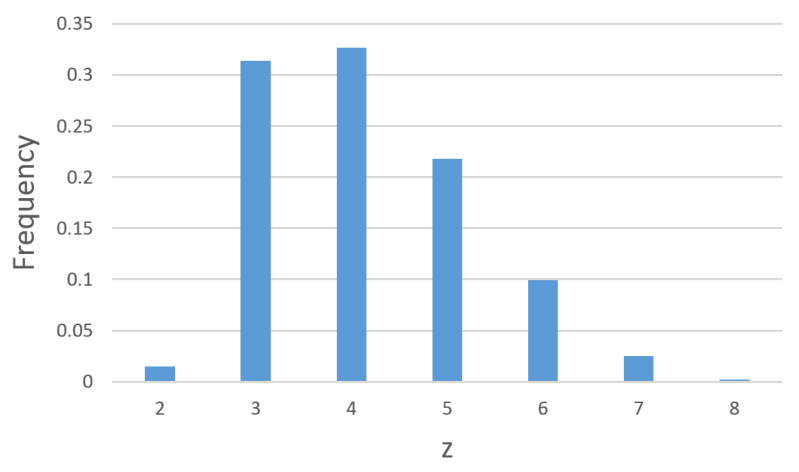

Figure 4. Distribution of the number of contacts $z$ of the removed grains.

Figure 4 shows the distribution of the number of contacts $z$ of the removed grains. We see that a majority of the removed grains have a contact number between $z=3$ and $z=5$, consistent with the average contact number for the entire system of $Z \simeq 4$.3. Only a very small number of the removed grains have a value of $z=2,7$ or 8 . This graph shows that by randomly removing grains from the system we are not uniformly sampling the possible contact numbers, but are instead sampling based on the distribution of contact numbers already present in the system.

To determine the effect of the contact number of the grain that is removed on the rearrangement of the system, in Figure 5 we plot the average movement of all grains within the packing $\left(D_{\text {avg }}\right)$ normalized by the radius of the grains $(R)$ against the contact number of the grain that was removed. The largest average movement of the grains in the sample occurs when a grain with $z=2$ is removed, with on average all grains moving by a distance equal to $1.7 \%$ of the grain radius. Note however, that as the data in this plot is an average over all of the grain removal events and as we have very few events with $z=2,7$ and 8 , there may be considerable random variation in the average values at these three points. Removing grains with $z=3$ leads to the smallest average movement of the sample $(0.25 \%$ of the grain radius) with a consistent increase in the average movement in the sample seen for larger contact numbers up to $z=7$. It is unclear for the decrease observed for $z=8$ if this is just due to being a single measurement point and hence highly susceptible to random variation.

Figure 6 shows the evolution of the packing fraction with the number of grains removed for the packing of ellipsoids with aspect ratio of 0.7 . In this case we start with a slightly lower packing fraction of $\phi \simeq 0.575$ and perform 800 iterations of removal of grains. We see a similar variation in the packing fraction as grains are removed to the spherical case (Figure 3), with fewer increases in packing fraction due to avalanche events. The packing fraction levels off at $\phi \simeq 0.548$ after 700 grains have been removed, slightly above the loosest packings that are obtained by sedimentation of $\phi \simeq 0.545$.

The packing fraction variation with number of grains removed shows the ability of this technique to transition

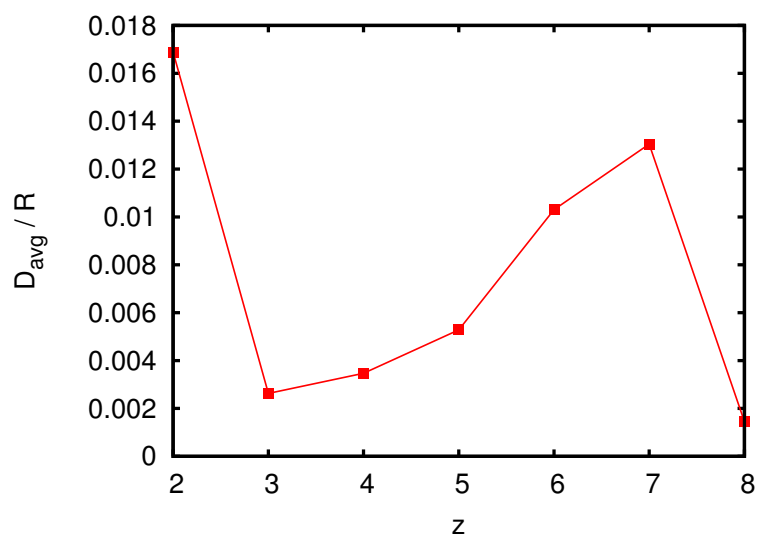

Figure 5. Variation in the average movement of a grain in the sample when a grain with a contact number of $z$ is removed. Note very few grains have a $z=2,7$ or 8 , meaning that considerable random variation in the value at these points would be expected.

between a broad range of packing fractions right down to close to the loosest mechanically stable packings obtained by sedimentation processes. However, one key concern is the degree of ordering that is being induced as a result of this technique, and whether we are obtaining packings with very different structural properties to those obtained via sedimentation. In particular here we will investigate the degree of orientational ordering of the ellipsoids and how this varies as we remove grains. We quantify the degree of orientational alignment using an order parameter

$$
\chi_{\theta}=\frac{3}{2}\left\{\frac{1}{N} \sum_{i=1}^{N} \cos 2\left(\theta_{i}-\frac{\pi}{2}\right)-\frac{1}{3}\right\}
$$

where $\theta_{i}$ is the angle between the semi-major axis of the $i$-th particle and the vertical axis [10]. If all grains have random orientations, $\chi_{\theta}=0$, while if the grains all lie flat $\chi_{\theta}=1$. We have also determined the more commonly used $S_{2}$ order parameter [11], quantifying the degree of orientational ordering of the major axes of the grains about their average nematic director, and have found that this parameter follows the same trends observed for $\chi_{\theta}$.

Figure 7 shows the variation of the angular alignment of the grains with the number of grains removed. The ellipsoids initially have a small degree of angular alignment with $\chi_{\theta} \simeq 0.145$. As more grains are removed and the packing fraction reduces, the degree of alignment only varies very slightly, with an increase to $\chi_{\theta} \simeq 0.16$, before plateauing. There is a steady increase in the degree of alignment after 450 grains have been removed, followed by a very sharp drop that exactly coincides with a sharp increase in packing fraction of $\Delta \phi=0.005$ when 500 grains have been removed. The degree of alignment then shows a consistent decrease to $\chi_{\theta} \simeq 0.13$ when 800 grains have been removed. The results clearly show that the reduction in packing fraction over the 800 grain removals is not due to any increase in ordering within the system, with the final orientational ordering being slightly lower than for the initial configuration. 


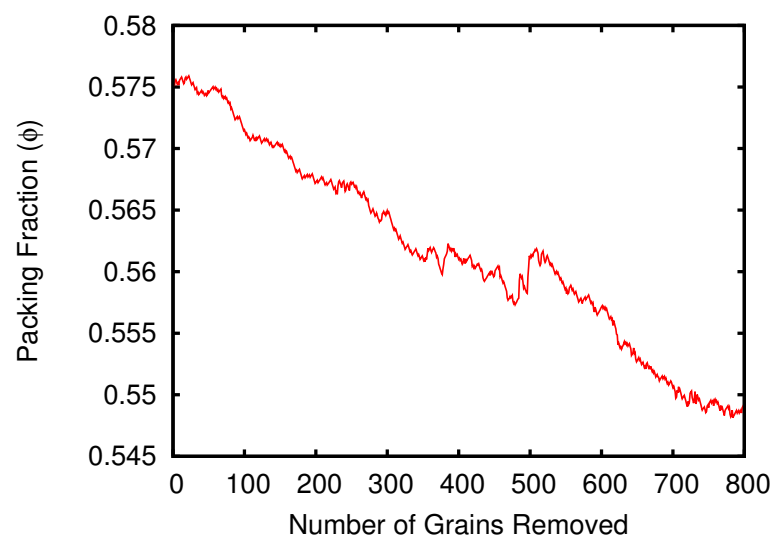

Figure 6. Evolution of the packing fraction as grains are iteratively removed from a packing of ellipsoids with an aspect ratio of 0.7 .

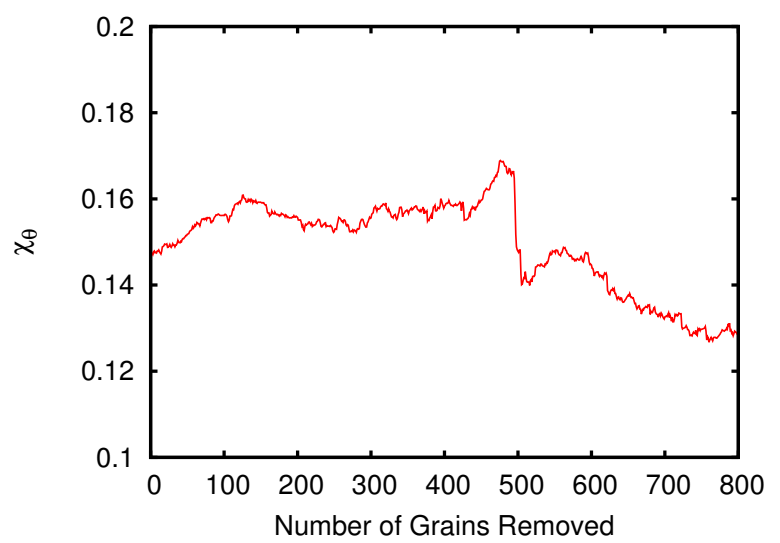

Figure 7. Evolution of the orientational order parameter $\chi_{\theta}$ as grains are iteratively removed from a packing of prolate ellipsoids with an aspect ratio of 0.7 .

\section{Conclusions}

We have presented results for a novel method for exploring the structure of random packings of granular matter at packing fractions between the loosest and densest mechanically stable random packings. We find that our technique of random removal of grains and relaxation of the sample allows us to study density variations for spheres down to packing fractions of $\phi \simeq 0.57$ and for ellipsoids down to $\phi \simeq 0.548$, close to the loosest mechanically stable packings obtained by sedimentation protocols. The technique also allows us to generate a continuous sequence of packings that ranges up to the densest packings. Our analysis of the effect on the structure of the sample of spheres from removing grains with different contact numbers shows the clear relationship between the contact number and the degree of rearrangement of the sample due to the removal of a grain. For the sphere system, we see an increase in the average displacement of the particles in the sample with the contact number of the removed grain for particle contacts from $z=3$ to $z=7$, representing the vast majority of the grains in the system. While our investigation of the orientational ordering in the sample of ellipsoids demonstrates that large variations in density can be achieved with this protocol without significant variations in the degree of orientational ordering within the system

The results presented here may be of particular interest in exploring more exotic granular structures and the very loose limits of mechanical stability. Understanding the degree of structural rearrangement from removing grains from the system may be especially useful in determining strategies for reducing the density of the system below the limits observed when generating granular packings using traditional techniques such as pouring.

\section{References}

[1] G.W. Delaney, P.W. Cleary, EPL (Europhysics Letters) 89, 34002 (2010)

[2] F.M. Schaller, M. Neudecker, M. Saadatfar, G.W. Delaney, G.E. Schröder-Turk, M. Schröter, Phys. Rev. Lett. 114, 158001 (2015)

[3] A. Baule, H.A. Makse, Soft Matter pp. 4423-4429 (2014)

[4] T. Aste, The Pursuit of Perfect Packing (Bristol and Philadelphia: IOP Publishing Ltd, 2000)

[5] P. Cundall, O. Strack, Geotechnique 29, 65, 47 (1979)

[6] P.W. Cleary, Engineering Computations 21, 169 (2004)

[7] P.W. Cleary, Powder Technology 179, 144 (2008)

[8] G.G. Pereira, P.M. Dupuy, P.W. Cleary, G.W. Delaney, Progress in Computational Fluid Dynamics 12, 176 (2012)

[9] G.W. Delaney, J.E. Hilton, P.W. Cleary, Physical Review E 83, 051305 (2011)

[10] B.J. Buchalter, R.M. Bradley, EPL (Europhysics Letters) 26, 159 (1994)

[11] A. Donev, S. Torquato, F.H. Stillinger, R. Connelly, Journal of Applied Physics 95, 989 (2004) 